\title{
Soziale Kommunikation und Wahrheitsverwaltung Überlegungen zu Aetatis novae Nr. 10 über die Medien im Dienst der kirchlichen Gemeinschaft
}

\section{von Helmuth Rolfes*}

\section{Zwei Medieninstruktionen ohne Zusammenhang?}

In relativ kurzem zeitlichem Abstand wurden in Rom zwei Medieninstruktionen veröffentlicht. ${ }^{1}$ Am 22.02.1992 erschien nach einem mehr als fünfjährigen Diskussionsprozeß der Mitglieder des „Päpstlichen Rates für die sozialen Kommunikationsmittel" und der entsprechenden internationalen Beratergremien endlich das lang erwartete Nachfolgedokument zu Communio et progressio: die Pastoralinstruktion „Aetatis novae“ zur sozialen Kommunikation zwanzig Jahre nach Communio et progressio.

Am 09.06.1992 brachte die Kongregation für die Glaubenslehre eine an die Bischöfe gerichtete „Instruktion über einige Aspekte des Gebrauchs der sozialen Kommunikationsmittel bei der Förderung der Glaubenslehre" heraus. Unterzeichnet mit Datum vom 30.03.1992 ist diese Instruktion vom Präfekten der Glaubenskongregation, Kardinal Josef Ratzinger. Sie enthält im wesentlichen eine Zusammenstellung aller relevanten kirchenrechtlichen Regelungen zum Bereich der sozialen Kommunikation, insofern die Glaubenslehre tangiert wird.

Während Aetatis novae nach eigenem Bekunden die Grundsätze und Einsichten aus den maßgeblichen Konzils- und Nachkonzilsdokumenten zur sozialen Kommunikation auf die heutige Situation anwenden und für die $\mathrm{Pa}$ storal ein Arbeitsinstrument sowie ein Mittel der Ermutigung an die Hand geben will (vgl. Nr. 1), hat die Instruktion der Glaubenskongregation eine andere Zielsetzung: Sie will die Bischöfe und höheren Ordensoberen, denen die „Aufgabe der Sorge und Überwachung" der sozialen Kommmunikationsmittel übertragen ist, insofern in ihnen Fragen der Glaubens- und der Sittenlehre behandelt werden, an jene Normen des kanonischen Rechtes erinnern, mit denen der rechtlich notwendige und mögliche Handlungsrahmen für diesen Bereich abgesteckt ist (vgl. Einführung).

Von dieser Zielsetzung her könnte man an sich die Instruktion aus der Glaubenskongregation als einen Text interpretieren, der lediglich längst Bekanntes aus dem CIC zur praktischen Handhabung für die Bischöfe noch einmal zusammenstellen will. So schätzt auch der Präsident des Päpstlichen Rates für die sozialen Kommunikationsmittel, Erzbischof John P. Foley, die Instruktion ein: „The 'Instruction on Some Aspects of the Use of the Instruments of Social Communication in Promoting the Doctrine of the Faith" is a useful summary of the norms of Church law on the subject of books and articles on Catholic doc-

Dr. Dr. Helmuth Rolfes ist Professor an der Gesamthochschule/Universität Kassel für Katholische Theologie (Schwerpunkt: Systematische Theologie). 
trinal or moral teaching either written by Catholics or published under Catholic auspices. The instruction is meant to guarantee the principle of "truth in packaging" - that is, that what is published as reflective of Catholic faith or morals may be certified to be so by those chiefly responsible for the preaching of the Gospel and the guidance of the faithful, the diocesan bishops. The instruction does not really enunciate new principles; it gathers existing norms in a brief document in which long valid principles are made more readily accessible." ${ }^{2}$

Sicher wird man nicht behaupten können, mit Aetatis novae und der neuen Instruktion aus der Glaubenskongregation solle die nach dem II. Vatikanischen Konzil und mit Communio et progressio eingeschlagene Linie in der Verhältnisbestimmung von Kirche und Medien verlassen werden. Entsprechende Befürchtungen hatte John P. Foley bereits im Vorfeld von Aetatis novae zu zerstreuen versucht. ${ }^{3}$ Daß mit einer solchen Gefahr jedoch durchaus zu rechnen war, zeigt die Stellungnahme des deutschen Medienbischofs Spital, der mit Bezug auf den deutschen Beitrag bei der Erarbeitung von Aetatis novae in einem KNA-Interview feststellte: „Wir haben in unserem Gutachten in erster Linie darauf geachtet, daß an keiner Stelle der Eindruck entstehen kann, als solle 'Communio et progressio' zurückgenommen werden, und außerdem haben wir auf die Form einer Pastoralinstruktion gedrungen. Damit sind wir auch durchgekommen. ${ }^{" 4}$

Nun entspricht es ohnehin nicht kirchenamtlicher Gepflogenheit, eine einmal bezogene Position direkt zu revidieren oder zu korrigieren. Kurskorrekturen werden durch bestimmte Akzentuierungen im Text, durch Verschweigen oder Betonen früherer Positionen oder durch eine gezielte Auswahl von Zitaten aus anderen kirchenamtlichen Dokumenten vorgenommen. Liest man unter dieser Rücksicht Aetatis novae und nimmt gleichzeitig auch die Instruktion aus der Glaubenskongregation hinzu, so lassen sich doch einige bedeutsame Verschiebungen gegenüber Communio et progressio feststellen. Ob ein politischer Zusammenhang zwischen Aetatis novae und der Instruktion aus der Glaubenskongregation besteht, läßt sich von außen nicht klären. Es bleibt aber zu fragen, warum fast zum gleichen Zeitpunkt, zu dem die Medieninstruktion aus dem Päpstlichen Rat für die sozialen Kommunikationsmittel erschien, gewissermaßen im Windschatten dieser Veröffentlichung, auch die Glaubenskongregation mit einer eigenen Instruktion aufwartet. Daß es sich nur um einen Zufall handelt, sollte man eigentlich ausschließen. Immerhin hatte die Glaubenskongregation vor der Veröffentlichung auch den Päpstlichen Rat für die sozialen Kommunikationsmittel konsultiert (vgl. Einführung).

Die Verbindung zwischen beiden Instruktionen läßt sich teilweise aufhellen, wenn man in Betracht zieht, daß in der Endredaktion von Aetatis novae bei dem vor einer Veröffentlichung eines Kirchendokumentes üblichen Durchlauf durch die römischen Instanzen (bes. Staatssekretariat und Glaubenskongregation) in der Nr. 10 einige Einfügungen vorgenommen wurden, die nicht im Entwurf zu Aetatis novae enthalten waren, der den Titel „Toward 2000" trug. Näherhin handelt es sich besonders um einen Verweis auf das kanonische Recht und um einen Bezug auf die 1990 von der Glaubenskongregation herausgebrachte Instruktion über die kirchliche Berufung des Theologen. Genau hier gibt es 
inhaltlich eine deutliche Verbindungslinie zur neuen Instruktion aus der Glaubenskongregation, die besondere Aufmerksamkeit verdient.

Der Einwand, diese Instruktion habe inhaltlich nichts mit der Massenkommunikation und den Massenmedien zu tun und trage deswegen einen irreführenden Titel, ist nur bedingt richtig. Richtig ist daran, daß es der Instruktion tatsächlich nicht um die Massenmedien im allgemeinen geht, sondern um bestimmte durch das Recht geregelte Fragen der innerkirchlichen Kommunikation und des Gebrauchs der Medien, besonders der Bücher, für diese Kommunikation, insofern sie Fragen des Glaubens und der Sittenlehre berühren. Wenn dennoch in der Instruktion sowohl im Titel als auch im Text von den sozialen Kommunikationsmitteln gesprochen wird, so ist damit erst einmal die Sprachregelung des CIC aufgenommen, die in Buch III De Ecclesiae munere docendi (Verkündigungsdienst der Kirche) einen eigenen Titulus IV „de instrumentis communicationis socialis et in specie de libris" (Soziale Kommunikationsmittel, insbesondere Bücher) aufführt. Die Instruktion stellt vor allem die Canones aus diesem Teil des CIC zusammen. Bereits die Überschrift im CIC macht deutlich, daß die Canones nicht nur über Bücher, sondern grundsätzlich auch über die sozialen Kommunikationsmittel insgesamt handeln. Wenn die Instruktion im eigenen Titel die Formulierung aus dem CIC „et in specie de libris" nicht aufnimmt, kann man darin eine Unbedachtheit oder eine Nachläßigkeit der Verfasser der Instruktion erblicken. Ob es sich wirklich nur darum handelt, muß die Interpretation der Instruktion ergeben.

\section{Soziale Kommunikation}

Für die soziale Dimension der Kommunikation sind die Medien, und dazu gehören in unserer modernen Welt ausdrücklich auch die Massenmedien, unverzichtbar. Aetatis novae zitiert Communio et progressio, wonach „die Kirche in den sozialen Kommunikationsmitteln 'Geschenke Gottes' erblickt, weil sie nach dem Ratschluß der göttlichen Vorsehung die Menschen brüderlich verbinden, damit diese im Heilswerk Gottes mitwirken“ (Nr. 22). Die aus diesem theologischen Ansatz mögliche positive Sicht der Medien begründete in der Zeit nach Communio et progressio eine konstruktive Neubestimmung des Verhältnisses von Kirche und Medien, die für die kirchliche Praxis noch längst nicht überall mitvollzogen wurde. ${ }^{5}$

Aetatis novae kündigt diese Sichtweise aus Communio et progressio nicht auf. Aber in der Einschätzung der tatsächlichen Medienmacht sich eine deutlich kritische Bewertung bemerkbar. So spricht Aetatis novae die in Verbindung mit der rasanten Entwicklung der modernen Kommunikationssysteme sich abzeichnenden weitreichenden kulturellen, sozialen, politischen und wirtschaftlichen Veränderungen an, die auch Anlaß zu Kritik und Besorgnis geben. Dieser Ansatz von Aetatis novae ist grundsätzlich zu begrüßen, da er zu einer realitätsbezogenen, nicht von theologischen Überhöhungen geleiteten Sicht der Medienwirk- 
lichkeit führt. Er sollte nicht mit dem Vorwurf einer pessimistischen Bewertung der Medien belegt werden.

Gerade weil Aetatis novae sich um eine kritische Mediensicht bemüht, erhält auch das Plädoyer für das Recht aller auf Information und Kommunikation ein besonderes Gewicht.

Was Aetatis novae zur Verteidigung des Rechtes auf Information und Kommunikation sagt, verdient insgesamt zitiert zu werden: „Es ist untragbar, daß die Ausübung der Kommunikationsfreiheit vom Reichtum, von der Erziehung oder von der politischen Macht abhängt. Das Recht auf Kommunikation ist ein Recht, das allen zusteht. Das erfordert besondere Anstrengungen auf nationaler und internationaler Ebene, nicht nur um den Armen und Machtlosen den Zugang zur Information zu geben, die sie für ihre individuelle und soziale Entwicklung brauchen, sondern auch um sicherzustellen, daß sie bei der Entscheidung über den Inhalt der Medien und bei der Festlegung der Strukturen und der Politik ihrer nationalen Kommunikationseinrichtungen tatsächlich eine verantwortungsvolle Rolle spielen. Wo rechtliche und politische Strukturen die Beherrschung der Massenmedien durch Eliten begünstigen, muß die Kirche nachdrücklich auf der Respektierung des Rechts auf Kommunikation und insbesondere auf ihrem eigenen Zugangsrecht zu den Medien bestehen, während sie gleichzeitig für ihre eigenen Mitglieder und für die Bevölkerung insgesamt nach alternativen Kommunikationsmodellen sucht. Das Recht auf Kommunikation gehört im übrigen zum Recht auf religiöse Freiheit, die nicht auf die Kultfreiheit beschränkt werden sollte" (Nr. 15).

Diese Passage aus Aetatis novae gehört sïcher zu den wichtigen Abschnitten, die der Medienpolitik der Kirche eine von einer hohen Wertschätzung der sozialen Kommunikation getragenen Richtung weist. Man wird den Forderungen nach voller Informations- und Kommunikationsfreiheit nur zustimmen können. Wie glaubwürdig kann die Kirche aber sein, wenn sie mit deutlichen Worten nach außen eine Freiheit der Kommunikation fordert, von der sie nach innen mit ganz anderen Worten redet?

Bei der Lektüre von Aetatis novae Nr. 10 läßt sich der Verdacht nicht abweisen, daß die sittlichen Maßstäbe, die für die soziale Kommunikation gelten und die der Gesellschaft insgesamt mit Recht vorgehalten werden, für die innerkirchliche Kommunikation nicht in gleicher Weise gültig sind. Schon in Communio et progressio zeigte sich nach G. Deussen an manchen Stellen ein solches gespaltenes Bewußtsein, das „manche entscheidende und fortschrittlich sich gebende Forderung an die allgemeine Öffentlichkeit im innerkirchlichen Bereich nicht verifiziert". ${ }^{6}$ Deussen sieht darin gånz allgemein eine Schwäche kirchlicher Dokumente, „die darin besteht, der Gesamtgesellschaft mit recht vernünftigen und notwendigen Forderungen aufzuwarten, während man im eigenen Bereich nicht nur die Anwendung, sondern auch die grundsätzliche Revision von Strukturen mit dem Hinweis auf die 'besondere Natur' der Institution Kirche schuldig bleibt. ${ }^{47}$

Auch Aetatis novae zeichnet sich in der Nr. 10 durch diese Schwäche aus, die durch die Instruktion aus der Glaubenskongregation noch vergrößert wird. 


\section{Das Recht auf Dialog und Information innerhalb der Kirche}

Aetatis novae erinnert zur Eröffnung des Abschnittes über den Dienst der Medien für die kirchliche Gemeinschaft „an das wichtige Recht auf Dialog und auf Information innerhalb der Kirche" (Nr. 10) und bezieht sich bei dieser Erinnerung auf Communio et progressio Nr. 114-121. Dort wird in einem eigenen Abschnitt über öffentliche Meinung und Kommunikation im Leben der Kirche festgestellt, „daß die Kirche der öffentlichen Meinung bedarf, die aus dem Gespräch ihrer Glieder erwächst“ (Nr. 115). Zwar kennt auch Communio et progressio den Verantwortungsbereich des Lehramtes in diesem Gespräch, dieser ist jedoch eingebettet in eine Theologie von der Kirche als Volk Gottes. Communio et progressio begreift die Meinungsfreiheit aller Katholiken deshalb auch aus dem Glaubenssinn, wie er der Gesamtheit der Gläubigen zukommt,und aus der Liebe, die die Kommunikationsfreiheit christologisch verankert. Jedem Gläubigen kommt eine wirkliche Meinungs- und Redefreiheit innerhalb der Kirche zu. Das schließt für Communio et progressio die Lehrautorität des Amtes nicht aus. Die Blickrichtung ist aber nicht vom Lehramt, dem die Sorge für die unverfälschte Bewahrung und Deutung der Glaubenslehre obliegt, zu den Gläubigen, die dann diese Glaubenslehre im Gehorsam empfangen, sondern eher umgekehrt: Vom Glaubenssinn der Gesamtheit der Gläubigen hin zum Lehramt, das innerhalb des Volkes Gottes eine besondere Dienstfunktion für die Bewahrung des Glaubens hat. Mit dieser Blickrichtung folgt Communio et progressio der Kirchenkonstitution des II. Vatikanums, auf die es sich auch ausdrücklich bezieht.

Gleichzeitig weiß Communio et progressio auch um den geschichtlichen Charakter aller Entscheidungsfindungen in Fragen der Wahrheit. Mit dem Hinweis auf die Geschichte soll nicht der beliebigen Deutung der Glaubenswahrheiten Tür und Tor geöffnet werden, wohl aber wird damit die Tatsache anerkannt, daß alle, solange sie treu zum Lehramt stehen, in der Kirche „das Recht und die Pflicht [haben] in Freiheit danach zu suchen, wie sie die geoffenbarten Wahrheiten tiefer verstehen und diese unter ständig sich wandelnden gesellschaftlichen Verhältnissen glaubwürdiger bezeugen können" (Nr. 116).

Für den Bereich des wissenschaftlichen Forschens der Theologen, auch dieser Bereich gehört ja zur innerkirchlichen Kommunikation, fordert Communio et progressio die notwendige Freiheit, in Artikeln und Büchern die Forschungsergebnisse austauschen zu können. Wenn dann bestimmte noch unausgereifte Thesen auch in den Massenmedien aufgegriffen werden, was hin und wieder unausweichlich geschieht, so sollen die Gläubigen befähigt werden, zwischen der in der Glaubensunterweisung vorgetragenen authentischen Lehre der Kirche und den oft vereinfachenden und teilweise auch verzerrenden Meinungsäußerungen in der Presse unterscheiden zu lernen. Grundsätzlich aber gilt: „Da die Entfaltung der öffentlichen Meinung in der Kirche lebensnotwendig ist, muß jeder Gläubige das Recht und die Möglichkeit haben, sich über alles zu informieren, was erforderlich ist, um im Leben der Kirche eine aktive Rolle zu übernehmen" (Nr. 119).

Communio et progressio bemüht sich auch innerkirchlich um einen angstfreien Umgang mit den Medien. Dem Gespräch und dem ungehinderten 
Informationsfluß wird hohe Priorität beigemessen. Dem Gläubigen, der mit den sozialen Kommunikationsmitteln umgeht, traut Communio et progressio zu, im Umgang mit der Vielzahl der Informationen und im Gespräch eine verantwortliche Position zu beziehen.

\section{Der Rückgriff auf das Kirchenrecht}

Abgesehen von der Erinnerung an diese wichtige Position zum innerkirchlichen Dialog in Communio et progressio bleibt in Aetatis novae zur gleichen Frage nichts mehr übrig. Hier geschieht mehr als nur eine Akzentverschiebung. Nachdem nämlich Aetatis novae an das wichtige Recht auf Dialog und auf Information innerhalb der Kirche mit Hinweis auf Communio et progressio erinnert hat, denkt es für den Schutz und die Förderung dieses Rechtes vor allem an die Weisung des kirchlichen Gesetzbuches und zitiert aus dem CIC Canon 212,2 und 3, "wonach es den Gläubigen im Gehorsam gegenüber den Hirten der Kirche 'unbenommen [ist], ihre Anliegen, insbesondere die geistlichen, und ihre Wünsche' diesen Hirten 'zu eröffnen', und wonach sie 'entsprechend ihrem Wissen, ihrer Zuständigkeit und ihrer hervorragenden Stellung das Recht und bisweilen sogar die Pflicht haben, ihre Meinung in dem, was das Wohl der Kirche angeht, den geistlichen Hirten mitzuteilen" (10).

Der zuvor mit Hinweis auf Communio et progressio bemühte innerkirchliche Dialog und das Recht auf Information und Dialog steht hier nun im Zusammenhang von Glaubensgehorsam und Gesetzesgehorsam als Grundpflicht des Gläubigen (CIC 212, 1), wobei auf dieser Basis dem Gläubigen das Recht dann zugestanden wird, die Hirten über ihre vor allem geistlichen Bedürfnisse und über ihre Wünschen zu unterrichten und je nach Qualifikation und Rang sogar die Pflicht vorhanden ist, ihre Ansichten bezüglich des Wohles der Kirche den Hirten respektvoll mitzuteilen. Immerhin nennt der Canon 212,3 auch noch das Recht bzw. in bestimmten Fällen auch die Pflicht, diese Meinungsäußerung in angemessener Art und Weise auch "den übrigen Gläubigen kundzutun“. Dieser Aspekt fältt in Aetatis novae aus. Der entsprechende 2. Teil des Canons 212,3 wird nicht mitzitiert.

Mit innerkirchlichem Dialog hat eine solche Regelung eigentlich nichts mehr zu tun und wie auf diesem Wege das Recht auf Information und Dialog für alle Gläubigen innerhalb der Kirche gefördert und geschützt werden soll, dürfte ebenfalls schwer zu vermitteln sein.

Die 2 und 3 des Canons 212 sind mit Nuancen wörtlich aus Lumen gentium Art. 37, Abs. 1 übernommen worden. Allerdings geht es in Lumen gentium nicht in erster Linie um einen Glaubens- und Gesetzesgehorsam gegenüber den Hirten, sondern um die wechselseitige Beziehung von Hirten und Laien. ${ }^{{ }^{\circ}}$ Der CIC ändert bereits diese Perspektive. So wundert es auch nicht, daß schon das Kirchenrecht nicht mehr die Aufforderung aus den Konzilstext mit aufnimmt, nach der die Gläubigen ,in Wahrhaftigkeit, Mut und Klugheit" den Hirten ihre Auffassung darlegen sollen. In Aetatis novae ist davon ebenfalls nicht die Rede. 
Aetatis novae meint sogar, daß durch das so beschriebene Recht auf respektvolle Kundgabe der eigenen Meinung in bestimmten Fällen die „Glaubwürdigkeit und Wirksamkeit der Kirche" aufrecht erhalten und gestärkt wird, und daß damit sogar ein Weg gewiesen wird, den in der Gemeinschaft der Dreieinigkeit verankerten Gemeinschaftscharakter der Kirche konkret zu verwirklichen. Im Kontext der vorherigen rechtlichen Rahmenvorgabe für den Dialog wirken solche theologischen Allgemeinplätze verhängnisvoll. Ihr ideologischer Gebrauch ist offensichtlich.

Auf der einen Seite wird ein streng hierarchisch orientiertes Kommunikationsmodell gefordert, auf der anderen Seite wird die gleiche Würde und Sendung aller Getauften hervorgehoben. Das kommt auch in folgenden Sätzen zum Ausdruck: „Zwischen den Mitgliedern dieser Gemeinschaft, die die Kirche darstellt, besteht eine grundlegende Gleichheit in Würde und Sendung, die ihren Ursprung in der Taufe hat und der hierarchischen Struktur und der Vielfalt der Aufgaben und Ämter zugrunde liegt. Diese Gleichheit soll in einer aufrichtigen und respektvollen Beteiligung an Information und Meinungsäußerung Ausdruck finden“ (Nr. 10). Hier wird das Recht auf Dialog und Information auf aufrichtige und respektvolle Beteiligung zurückgeschraubt, obwohl der theologische Satz zuvor eigentlich eine andere Akzentsetzung erwarten ließ.

Wie in den kirchenamtlichen Selbstdefinitionen der letzten Jahre insgesamt, so wird auch in Aetatis novae an dieser Stelle sichtbar, wie das Verständnis der Kirche als Communio zunehmend in hierarchischen und rechtlichen Vorstellungsmustern ausformuliert wird. ${ }^{9}$

\section{Meinungsverschiedenheiten und irrige Gedanken}

Wichtiger als das Recht auf Dialog und Information, auf Partizipation aller am Prozeß der Meinungsäußerung scheint gegenwärtig ohnehin die Frage zu sein, wie man innerkirchlich mit Meinungsverschiedenheiten umgehen soll. Hier bemüht Aetatis novae zwei Zitate aus der 1990 von der Kongregation für die Glaubenslehre verfaßten Instruktion über die kirchliche Berufung des Theologen: „Im Fall von Meinungsverschiedenheiten muß man im Auge behalten, daß 'man nicht durch das versuchte Ausüben von Druck auf die öffentliche Meinung zur Klärung von Lehrfragen beitragen und der Wahrheit dienen wird'. In der Tat, 'nicht alle Ideen und Vorstellungen, die im Volk Gottes im Umlauf sind, lassen sich rundweg mit dem 'Glaubenssinn' gleichsetzen'“ (Nr. 10).

Ein ähnliches Problem bewegte vermutlich auch die Glaubenskongregation mit ihrer jüngsten Instruktion. In der Einführung heißt es nämlich, daß „durch die sozialen Kommunikationsmittel im allgemeinen und durch die Bücher im besonderen heute immer mehr irrige Gedanken verbreitet" werden. Nachdem bereits vom Standpunkt der Lehre in der auch von Aetatis novae zitierten Theologeninstruktion die Verantwortung der Hirten in Fragen des authentischen Lehramtes erläutert worden sei, solle nun gesagt werden, wie diese Verantwortung im Rahmen des Rechts wahrgenommen werden muß. 
Das Problem, das die Glaubenkongregation zur Herausgabe dieser Instruktion veranlaßt hat, ist leicht anschaulich zu machen: Theologen wie beispielsweise Leonardo Boff oder Eugen Drewermann erzielen mit ihren Büchern hohe Auflagen und erreichen damit eine Öffentlichkeit, die weit über theologische Fachkreise hinausreicht. Die Massenmedien verfolgen mit größter Aufmerksamkeit den Konflikt der Theologen mit der Kirche und bieten ihnen immer wieder auch eine Plattform an, diesen Konflikt und ihre eigenen Thesen in den unterschiedlichsten Facetten öffentlich zu machen.

Die Glaubenskongregation interpretiert solche Vorgänge als Versuch, „durch das versuchte Ausüben von Druck auf die öffentliche Meinung“ Lehrfragen klären zu wollen. Sie übersieht dabei aber, daß ohne die Massenmedien in unseren modernen Gesellschaften überhaupt keine öffentliche Meinung entstehen und artikuliert werden könnte und zieht deshalb auch die falschen Schlüsse. Anstatt sich selbst in einen Diskurs hineinzubegeben und auf die Kraft der eigenen Argumente, mit denen ja schließlich die authentische Lehre und Wahrheit vertreten werden soll, zu vertrauen, wird eine Bedrohungsfront aufgebaut. Das Volk Gottes, die Gläubigen begegnen in diesem Kontext als eine fürsorglich vom Lehramt zu beschützende und zu leitende Größe, in der ohnehin Ideen und Vorstellungen im Umlauf sind, die nicht dem „Glaubenssinn" entsprechen. Das Zutrauen in die Gläubigen, sich im Meinungsstreit auch eine verantwortliche eigene Meinung bilden zu können, ist in der Glaubenskongregation außerordentlich schwach ausgebildet.

Gegenüber der Konzils- und Nachkonzilszeit ist hier ein deutlicher Gesinnungswandel festzustellen. Er verbindet sich mit der Vorstellung, sog. irrige Gedanken durch Rückgriff auf das Recht und durch gewissenhafte Anwendung der kanonischen Normen zu verhindern, zumindestens aber sie nicht öffentlich werden zu lassen. Hätte das kirchliche Recht immer und überall gewissenhafte Anwendung gefunden und wäre befolgt worden, so wären nach dieser Logik sicher eine Reihe umstrittener Bücher gar nicht erst publiziert worden. Die Instruktion ermahnt die Bischöfe deswegen an ihre Aufsichtspflicht und führt dabei vor allem die Canones 822-832 an, die sich auf die sozialen Kommunikationsmittel und besonders auf die Bücher beziehen. ${ }^{10}$ Diese Canones sind neu in das Kirchenrecht aufgenommen worden. Im alten CIC gab es lediglich die Regelung zum Imprimatur (vorbeugende Bücherzensur cann. 1385-1394 CIC 1917) und zum Index der verbotenen Bücher (unterdrückendes Bücherverbot cann. 1395-1404 CIC 1917). Imprimatur und Index sollten dem Schutz von Glaube und Sitte dienen und gleichzeitig auch ein Instrument zur Disziplinierung an die Hand geben, das freilich, wie die Geschichte zeigt, häufig mißbraucht wurde. Das Bücherverbot erstreckte sich nämlich auf weite Bereiche und bezog später auch alle Printmedien und den Film mit ein. "Hier war mit einem juridischen Zaun ein katholischen Ghetto umgrenzt, das abwehrend alle 'schlechte Presse' und alles böse Schrifttum von Katholiken fernhalten sollte". " Das Projekt der Ghettoisierung des katholischen Bewußtseins ist gründlich gescheitert und wurde vom II. Vatikanischen Konzil beendet. So wurde das Bücherverbot (Index) 1966 aufgehoben. Auch das neue Kirchenrecht will diesen Weg nicht gehen. Die bei den Bischöfe einzuholende Druckerlaubnis (Approbation bzw. Erlaubnis) bezieht sich verpflichtend nur auf Ausgaben der Heiligen Schrift, auf liturgische 
und kirchenamtliche Texte, Gebetbücher, Katechismen sowie Bücher für den Religionsunterricht. Eine solche Regelung ist durchaus sinnvoll und sollte ernsthaft auch nicht bestritten werden. Wenn es in der Rechtsinstruktion nur um dieses Problem ginge, wäre die Instruktion selbst eigentlich überflüssig.

Strittig dagegen, und hier scheint das Problem der in der Einführung bezeichneten "irrigen Gedanken“ anzusiedeln zu sein, ist aber die Deutung des Canons 827,3 über die Schriften, für die das Urteil des Ortsordinarius angezeigt ist. Dort heißt es: „Es wird empfohlen, Bücher, die in 2 genannte Materien [Heilige Schrift, Theologie, kanonisches Recht, Kirchengeschichte, andere religiöse oder moralische Fragen, H. R.] behandeln, auch wenn sie nicht als Texte bei der Unterrichtserteilung benutzt werden, ebenso Schriften in denen etwas enthalten ist, was sich in besonderer Weise auf die Würde von Religion oder Sitten bezieht, dem Urteil des Ortsordinarius zu unterwerfen."

In einer engen Auslegung könnte man im Zusammenhang des Canon 827,1 und 2 vor allem an Begleitmaterialien für Religionsunterricht und Katechese sowie für die katholische Erziehung insgesamt denken. Der Abschnitt 3 läßt aber auch eine weitere Interpretation zu. Dann wird man an das weite Spektrum theologischer Schriften denken, zumal wenn sie sich von der Publikationsform her an eine größere theologisch und kirchlich interessierte Öffentlichkeit richten. Übrigens kann der Bischof, wie die Instruktion es im Sinne einer die weitere Interpretation des Canons 2273 stützenden Weise ausdrücklich bemerkt, „wann immer er besondere und spezifische Gründe hat, auch durch Befehl im Einzelfall (can 49) fordern, daß die genannten Schriften seinem Urteil unterworfen werden. Der Bischof kann Vorschriften für besondere Fälle „sowohl einzelnen Personen als auch Gruppen von Personen“ auferlegen. Dazu zählen z. B. Kleriker, Ordensleute, katholische Verlage. Auch für bestimmte Materien kann der Bischof eine entsprechende Vorschrift verfügen.

Bücher wie Leonardo Boffs „Kirche: Charisma und Macht“ oder Eugen Drewermanns „Die Kleriker" würden ein solches Verfahren wohl kaum bestanden haben, hätte der zuständige Ortsbischof durch Befehl in diesem Falle die Vorlage der Schrift vor der Veröffentlichung zur Überprüfung angefordert. Da Kleriker und Ordensangehörige dem Bischof bzw. dem Ordensoberen in besonderer Weise Gehorsam und Ehrerbietung schulden, müßte das Problem „irriger Gedanken" eigentlich in den Griff zu bekommen sein, - wenigstens in der Theorie. Daß ein betroffener Theologe oder ein katholischer Verlag die Möglichkeit hat, über ein Lehrbeanstandungsverfahren oder den Rekurs das bischöfliche Urteil überprüfen zu lassen, ändert nichts an der grundlegenden Situation.

\section{Wahrheitsverwaltung}

Das Recht auf freie öffentliche Meinungsäußerung innerhalb der Kirche, das durch Canon 212,3 geregelt werden sollte, ist durch die Verpflichtung zur Wahrung der Unversehrtheit des Glaubens und der Sitten, durch Ehrfurcht gegenüber den Hirten und durch die Beachtung des Allgemeinwohls sowie der Würde der Person begrenzt. Problematisch ist diese Begrenzung nicht an sich. Sie könnte, recht verstanden, sogar zu einer besonderen Ethik kirchlicher Kom- 
munikation führen. Problematisch ist sie aber wohl in Verbindung mit dem geforderten und im Canon 212,3 zum Ausdruck gebrachten kirchlichen Kommunikationsmodell, das einen lebendigen Austausch im Volkes Gottes durch die Forderung nach Gesetzes- und Glaubensgehorsam auf Seiten der Gläubigen einerseits und andererseits durch den Anspruch des „Besitzes“ der Wahrheit durch das Lehramt erst gar nicht zuläßt.

Die Frage der Wahrheitsfindung reduziert sich damit auf ein Autoritätsproblem. Daß auch die Kirche die Wahrheit nicht in vollendeter Gestalt besitzt, sondern im geschichtlichen Zeugnis Jesu Christi, daß sie bei der Wahrheitsfindung immer auch auf die Bezeugung des Glaubens an Jesus Christus durch die Gläubigen verwiesen bleibt und dieser Bezeugung zu dienen hat, wird nicht bedacht. Deshalb geschieht die Bewahrung und Vermittlung von Wahrheit auch nicht in kommunikativen Handlungszusammenhängen, sondern als Mitteilung von oben nach unten.

„Warum legt die Kirche solches Gewicht auf die Verantwortung ihrer Hirten, die Wahrheit zu vermitteln und den Gläubigen heranzubilden, dasselbe zu tun?", fragt Aetatis novae und gibt darauf sofort die verblüffende Antwort: „Der Grund dafür ist, daß das Gesamtverständnis dessen, was Kommunikation in der Kirche bedeutet, auf der Erkenntnis beruht, daß das Wort Gottes Ihn selbst mitteilt" (Nr. 10). Hier wird die Selbstmitteilung Gottes in seinem Wort in so enger Weise mit dem Anspruch des Lehramtes, die Wahrheit zu haben, zu verwalten und mitzuteilen verknüpft, daß die Mitteilung der Wahrheit durch das Lehramt an die Gläubigen am Ende selbst schon zum Grundmodell der Kommunikation in der Kirche wird. Zwischen diesem Kommunikationsverständnis und dem, was Communio et progressio über den innerkirchlichen Dialog und besonders auch über Christus als den großen Kommunikator und als Vorbild für diesen Dialog gesagt hat, liegen Welten.

In Aetatis novae und noch unmißverständlicher in der Instruktion aus der Glaubenskongregation setzt sich eine Mentalität durch, die für die Kommunikation innerhalb der Kirche auf kirchliche Autorität und auf Amtsvollmacht setzt, auf Durchsetzung von Wahrheitsansprüchen und nicht auf Überzeugung. Im Vordergrund steht, wie schon in der Theologeninstruktion klar ausgesprochen, ein Selbstverständnis des Lehramtes als einer von Gott selbst mit dem Charisma der Unfehlbarkeit ausgestatteten Instanz zur Sicherung und Verwaltung der Wahrheit in einem umfassenden Sinn. ${ }^{12}$ Für Kommunikation und Dialog bleibt nur wenig Raum. Daß das Lehramt auch eine Verpflichtung zum genauen Hinhören und Hinschauen auf die Zeichen der Zeit sowie auf das gelebte Glaubenszeugnis in der Kirche hat, kommt überhaupt nicht in den Blick. Um solches Hinhören und Hinschauen hatte Communio et progressio sich noch ausdrücklich bemüht. Von daher müßte eigentlich gerade auch das Lehramt in der Kirche Christus, dem „perfectus communicator“, im Verhalten ähnlich werden, wie Bernhard Häring es bereits vor 20 Jahren in einer theologischen Überlegung zu Communio et progressio forderte: „Eine nicht vom Hören geprägte und darum von den geistigen Strömungen und der Kommunikationsweise der Zeit distanzierte 'lehrende Kirche' liegt nicht auf der richtigen Wellenlänge. "' ${ }^{13}$ Häring hatte damals die Befürchtung geäußert, daß sich im Verhältnis der Kirche zur öffentli- 
chen und zur innerkirchlichen Kommunikation eine Polarisierung einstellen könnte, wenn einerseits die Kirche auf der medienpolitischen Linie von Communio et progressio im gesellschaftlichen Bereich eine starke öffentliche Meinung und eine weitere Entfaltung der sozialen Kommunikation fordert, „wenn aber andererseits an einem veralteten Modell der Theologie und ihres Verhältnisses zum Lehramt und zur Öffentlichkeit festgehalten und deshalb versucht würde, die als so wichtig anerkannte öffentliche Kommunikation 'unter Kontrolle' zu bringen " ${ }^{14}$ Die Nr. 10 aus Aetatis novae trägt sicher nicht dazu bei, diese von Häring befürchtete Polarisation abzubauen. Sie ist eher ein Beleg dafür.

Leider stehen auch die Aussagen zur Evangelisation in Aetatis novae im Horizont dieses Verständnisses einer lehrenden und wahrheitsverwaltenden Kirche. Folglich werden die Medien auch als "Werkzeuge“ angesehen, den Plan der Kirche zur Re-Evangelisierung bzw. Neuevangelisierung in der heutigen Welt zu verwirklichen (vgl. Nr. 11). Die Medien werden zu Instrumenten, mit denen der Welt die Wahrheit mitgeteilt wird. Ein solches Evangelisationskonzept ist zum Scheitern verurteilt. Es übersieht, daß Evangelisation sich theologisch vorrangig an die Kirche selbst richtet. Die Kirche ist der erste Adressat der Evangelisierung. Solange innerkirchliche Kommunikation aber durch eine Mentalität amtlicher Wahrheitsverwaltung dominiert wird, wird es schwer sein, Kirche als diesen ersten Adressaten der Evangelisierung zu verstehen. Dieses Evangelisationskonzept wird aber nicht nur scheitern, weil es an der theologischen Engführung partizipiert, wie sie in Nr. 10 von Aetatis novae zu erkennen ist; es wird auch scheitern, weil es eine längst überwunden geglaubte Instrumentalisierung der Medien als Möglichkeit für die soziale Kommunikation unterstellt.

Ob ein Pastoralplan, wie er im Anhang zu Aetatis novae als Rahmenvorgabe für die Ortskirche aufgestellt ist, wirksam und fruchtbar wird, hängt nicht zuletzt davon ab, ob jene die soziale Kommunikation letztendlich strangulierende Mentalität von amtlicher Wahrheitsverwaltung zugunsten einer echten Dialogbereitschaft überwunden wird, die die gieiche Würde und Sendung aller Getauften ernst nimmt.

\section{Anmerkungen}

1 Beide Instruktionen sind in der Reihe Arbeitshilfen, hg. v. Sekretariat der Deutschen Bischofskonferenz, Kaiserstr. 163, Bonn, 1992 veröffentlicht worden.

2 Response of Archbishop John P. Foley, President, Pontifical Council for Social Communications, to request by CNS for statement on recent instruktion from the congregation for the doctrine of the faith, Tuesday, June 2, 1992.

3 Vgl. O. Fuchs, Kirche und Medien auf dem Weg zum Jahr 2000, in: StZ 116 (1991) 411.

4 KNA - ID 13/26.03.1992, 7.

5 Vgl. M. Schmolke, Braucht die Kirche eine neue Medienpolitik?, in: CS 23 (1990), $197-$ 206.

6 G. Deussen, „Communio et Progressio“ auf dem Hintergrund der päpstlichen Lehrtradition, in: Kirche und Publizisitik, Beiheft $1 \mathrm{zu}$ Communicatio Socialis, Paderborn 1972, 34 .

7 Ebd.

$8 \mathrm{Vgl}$. F. Klostermann, Kommentar zum IV. Kapitel von Lumen Gentium, in: LThK, Das Zweite Vatikanische Konzil I, Freiburg-Basel-Wien 1966, 280-283.

9 Als jüngstes Beispiel für diese Tendenz in der Ekklesiologie läßt sich verweisen auf 
das von Kardinal Ratzinger mit Datum vom 28.05.1992 unterzeichnete „Schreiben an die Bischöfe der katholischen Kirche über einige Aspekte der Kirche als Communio".

$10 \mathrm{Vgl}$. zum folgenden den Kommentar von $\mathrm{H}$. Mussinghoff zu cann. 822-832, in: Klaus Lüdicke (Hg.), Münsterischer Kommentar zum CIC, Münster $1985 \mathrm{ff}$.

11 Ebd.

12 Vgl. H. Rolfes, Lehramt, Theologie und Öffentlichkeit, in: CS 23 (1990) 207-216.

13 B. Häring, Theologie der Kommunikation und theologische Meinungsbildung, in: Kirche und Publizistik, a. a. O., 39.

14 Ebd., $39 f$.

\section{SUMMARY}

The new pastoral instruction „Aetatis novae" has not taken back or canceled anything from "Communio et progressio". This is the official interpretation of the New media document. This interpretation does not stand up to critical examination, especially when taking into account the instruction published at the same time by the congregation of faith which lists the currently operative law of $\mathrm{CIC}$ in dealing with the instruments of social communication. One has to make a positive note of the realistic view of the mass media in "Aetatis novae" in which theological excessiveness is avoided. In "Aetatis novae" one can note a decisive shift of emphasis in comparison with "Communio et progressio" as regards the statements on inner-ecclesiastical communication. In this sphere the statements of "Aetatis novae" come close to certain statements of the instruction of the congregation of faith as regards the collected legal regulations. Communication is reduced to the communication of the truth from top to bottom which, when in doubt or in conflict, is to be achieved with the means of the CIC. The background of this completely one-sided model of communication is formed by the understanding of a church that teaches and administers the truth. The concept of evangelization for which the instruments of social communication are, according to „Aetatis novae“, insdispensable nowadays, is also determined by this ecclesiological narrowness.

\section{RÉSUMÉ}

La nouvelle instruction pastorale „Aetatis novae“ n'a ni contredit ni aboli "Communio et progressio“. C'est l'interprétation officielle du nouveau document de média. Cette interprétation ne résiste pas à un vérification critique, surtout si l'on tient comple de l'instruction de la Congrégation de Foi publiée en même temps qui rassemble le droit en vigueur concernant les instruments de la communication sociale.

Il faut louer l'image réaliste des mass médias en „Aetatis novae" qui évite des surélévation téologiques. Dans les énoucés sur la communication à l'intérieur de l'église en "Aetatis novae" on voit un changement décisif vis-à-vis de "Communio et progressio“. Dans le domaine les énoncés de „Aetatis novae“ s'approchent de certains énoncés de l'instruction de la Congrégation de Foi avec le receuil de lois. La communication se réduit à une transmission de vérité de haut en bas qui, dans le cas de doute ou de conflit, doit être exécutée à l'aide du droit canonique. Au fond de ce modèle de communication tout à fait unilatéral se trouve l'image d'une église qui enseigne et qui possède la vérité. Aussi la 
conception de l'évangélisation pour laquelle les instruments de la communication sociale selon "Aetatis novae" sont indispensable aujourd'hui est déterminée par cette étroitesse ecclésiologique.

\section{RESUMEN}

La nueva instrucción pastoral "Aetatis novae“ no le ha quitado nada a "Communio et progressio", ni tampoco la ha derogado. Esta es la interpretación oficial de este nuevo documento sobre los medios. Esta interpretación no resiste un análisis crítico, sobre todo si se toma en consideración la nueva instrucción, publicada en el mismo tiempo por la Congregación de la Fé, que reune las reglas del derecho vigente (CIC) en el trato con los instrumentos de la comunicación social. Se ha anotar como positivo en la "Aetatis novae" la visión realista de los medios de masas en los que se evitan las "demasías“ teológicas. Un cambio de acento decisivo, respecto a "Communio et progressio“, se encuentra en „Aetatis novae" en la declaración sobre comunicación en el interior de la iglesia. En este campo coinciden las declaraciones de „Aetatis novae“ con algunas de las de la Congregación de la Fé con las prescripciones de derecho reunidas. La comunicación se reduce a la notificación de la verdad de arriba hacia abajo, la que, en caso de duda o de conflicto, se impondrá utilizando los medios del derecho canónico. En el fondo de este unilateral modelo de comunicación se encuentra la concepción de una iglesia enseñante y adminstradora de la verdad. De esta forma, el concepto de evangelización, para el que según "Aetatis novae" los medios de comunicación son hoy en día irrenunciables, se encuentra determinado por esta estrechez de la jeraquía. 